\title{
Les liaisons @nodines : un imaginaire du roman e-pistolaire
}

\author{
Sophie Marcotte \\ Université Concordia
}

Les recherches sur la correspondance et le roman épistolaire, qui relèvent plus largement de ce qu'on pourrait appeler les discours de l'intime dans le domaine de la recherche littéraire, ont été fort nombreuses au cours des dernières décennies. Si plusieurs types de textes épistolaires ont suscité l'intérêt de la critique (lettre familière, lettre publique, lettre d'amour, lettre d'écrivain ou d'artiste, roman épistolaire, par exemple) et ont fait l'objet de nombreux travaux universitaires, tant d'un point de vue poétique, sémiologique et thématique que sociologique, 
la forme dérivée de ce dernier, le roman e-pistolaire (ou roman par courriels), apparue dans les années 1990 mais qui s'est multipliée dans les années 2000, reste encore peu commentée par les chercheurs. De même, de nombreux romanciers, depuis une quinzaine d'années, insèrent un ou des courriels à leur trame narrative ${ }^{1}$. Or, selon Benoît Melançon, « le courriel, forme d'expression aussi aisément sécable que la lettre avant lui, est devenu un ressort convenu de la fiction, au même titre que celui-ci avait pu s'approprier, au moment de leur apparition, le télégramme, le téléphone ou la télécopie » (2010, p. 3).

Ce seront les romans entièrement rédigés en mails que nous interrogerons ici. Si on voulait en établir un portrait général, on pourrait affirmer qu'il en existe essentiellement deux types. Le premier est le roman e-pistolaire qu'on qualifierait de "sentimental », dont l'intrigue se déploie autour d'un échange de mails qui devient le principal ressort de la relation - ou de la rupture - amoureuse. On peut citer l'exemple du roman à succès Quand souffle le vent du nord de Daniel Glattauer, paru en 2006 dans sa version originale en allemand et traduit en français en 2010, dont l'intrigue repose sur un échange de courriels entre un homme et une femme qui ne se connaissent pas et qui deviennent amoureux en s'écrivant, sans jamais se rencontrer. La relation se construit donc par un jeu de séduction qui passe exclusivement par les voies de communication numérique. P.S. He's Mine!, qui raconte l'histoire d'un triangle amoureux formé de deux jeunes adolescentes qui se sont rencontrées dans un camp d'été et du jeune homme dont elles sont devenues amoureuses et qui se plaît à

\footnotetext{
1 On pense entre autres au roman Scrapbook de Nadine Bismuth (2004), dont tout un chapitre est rédigé sous la forme d'un échange de courriels.
} 
fréquenter l'une et l'autre en même temps, représente un autre exemple de roman dont l'essentiel tourne autour de la thématique amoureuse (Rushton \& Schindler, 2000). Le second type est le roman d'entreprise (qu'on pourrait aussi appeler "roman corporatif»). La plupart des exemples ont paru en Grande-Bretagne et aux États-Unis. L'intrigue a généralement pour toile de fond le quotidien dans les grandes entreprises, les relations hiérarchiques, entre patrons et employés; et horizontales, entre employés, avec les imbroglios que cela peut engendrer. C'est à ce second type de roman, qui offre ainsi une représentation de l'entreprise moderne depuis la perspective des ressources humaines et des interactions entre les personnages, que l'analyse qui suit, fondée sur les romans $E$ (2000), E2 (2009) et Eleven (2006), sera consacrée. Le Baiser électrique de Martina Wachendorff (2001) est d'une certaine manière un composite des deux types de romans e-pistolaires évoqués. Il présente en effet le récit de la vie d'un couple et de ses enfants sur un yacht, où tous les individus communiquent entre eux (d'une cabine à l'autre!) et avec le reste du monde par courrier électronique. À la fois roman sentimental et roman d'entreprise, parce que les intrigues amoureuses qui se superposent, en superficie, cachent une trame beaucoup plus dramatique, celle d'un possible accident nucléaire, le roman de Wachendorff met en lumière un des problèmes fréquemment rencontrés dans les échanges de mails : l'envoi de messages par erreur ou volontairement (en copie cachée) à des gens à qui ceux-ci ne sont pas destinés.

Il ne s'agira pas ici, il faut le préciser, de commenter systématiquement les ressemblances et les différences au plan formel entre le roman épistolaire traditionnel et le roman par mails. Deux explications paraissent néanmoins s'imposer avant 
d'entrer dans le corpus à proprement parler. D'abord, comme l'illustre d'ailleurs Mobile Lovers de Banksy (2014), qui montre deux amoureux enlacés paraissant davantage intéressés par leur cellulaire que par l'étreinte qu'ils partagent et par l'éventualité d'un baiser, le courriel s'inscrit, peut-être encore plus que la lettre traditionnelle, dans une dynamique paradoxale de distance et d'absence, ce que Vincent Kaufmann, dans un essai paru en 1990 abondamment cité et consacré, notamment, à la correspondance de Flaubert, de Kafka, de Valéry et de Proust, a résumé par l'expression «équivoque épistolaire ». Écrire des courriels (en temps presque réel), en effet, permet de se rapprocher de l'autre, voire de l'étreindre, pour revenir à l'esprit du pochoir de Banksy, de garder le «canal» de communication constamment ouvert, tout en maintenant ce même autre à distance. Par ailleurs, l'éclatement, dans le courriel, des codes épistolaires traditionnels (forme plus relâchée, ton et niveau de langue familiers, manière de se représenter, etc.) contribue à faire basculer le roman epistolaire, en apparence du moins, dans la légèreté, la banalité, l'anodin, voire le vide. À partir de ces deux constats, on tentera notamment de voir ici de quelle manière tout cela - c'est-à-dire l'incommunicabilité et la banalité - finit par s'agencer pour former de véritables romans.

\section{@courriel}

Le courriel, mode de communication asynchrone et à distance, est devenu, on le sait, un outil incontournable dans toute entreprise ou milieu de travail modernes. Les nouveaux dispositifs mobiles ajoutent même une dimension d'ubiquité à cette pratique. On consulte désormais ses courriels sur des 
tablettes ou des portables, jour et nuit, au resto, pendant la leçon de violon de fillette ou le match de hockey de fiston. On s'en sert même pour communiquer avec des collègues qui occupent pourtant les bureaux voisins du nôtre. «Dans l'entreprise moderne », précise Fernando A. A. Lagraña (s.d.), «les conversations interpersonnelles, face à face, perdent de leur prépondérance au profit de communications virtuelles. » Or, cela améliore-t-il pour autant la productivité? La qualité des échanges? Les relations entre patrons et employés? L'utilisation qui est faite du courriel (l'analyse des romans choisis ici permettra d'ailleurs d'en faire la démonstration) montre bien qu'il sert tout autant, sinon plus, à perdre du temps qu'à en gagner.

Le courriel est en outre associé à une surcharge d'information. Son usage et le fait que l'on puisse désormais consulter sa messagerie sur des appareils mobiles fait ressortir un paradoxe intéressant par rapport à la frontière entre vie professionnelle et vie personnelle. En effet, alors que l'un des avantages $\mathrm{du}$ courriel par rapport aux autres types d'interaction se situe dans la possibilité de pouvoir y répondre n'importe où et, surtout, n'importe quand, la surabondance de messages qui en résulte a pour effet de brouiller les rôles et les fonctions de l'individu dans les sphères du travail et de la vie privée, tant dans l'espace que dans le temps : quand commence et quand se termine désormais une journée ouvrable? En outre, les mails donnent lieu à des comportements parfois déplacés (envoi d'émoticônes dans des messages adressés à des individus faisant partie de la haute direction d'une entreprise, par exemple), à des messages compromettants envoyés par erreur à des individus en position d'autorité, à l'envoi massif par certains collègues de messages mettant leurs réalisations 
du jour en valeur, à des transferts de messages à l'insu des destinateurs, à la circulation de rumeurs, voire à l'annonce de licenciements (qui exigeraient pourtant une conversation réelle) et au harcèlement de la part de certains correspondants qui exigent une réponse dans les minutes qui suivent l'envoi de leur message.

Parfois, l'échange de courriels donne aussi l'impression d'une conversation orale en différé tant il reproduit, par le ton, la syntaxe et le choix du vocabulaire, l'interaction en temps réel. Non seulement s'y exprime-t-on souvent sur un ton qu'on n'oserait jamais employer dans une conversation avec son interlocuteur, mais il en est venu à remplacer lesdites conversations, à un point tel que, lorsque les serveurs des institutions sont en panne ou que les fournisseurs Internet annoncent une interruption de service pour quelques heures, le temps de réaliser des travaux de maintenance sur les serveurs et les réseaux, les usagers se sentent démunis et oublient qu'il existe encore d'autres manières de communiquer avec autrui, comme le rappelle non sans ironie l'un des personnages des romans à l'étude :

Due to an ongoing software problem, e-mail will be shut down from 7:00am tomorrow. The repairs should be finished by the end of the day. IT apologises for the inconvenience. However, there is always the telephone. Alternatively, you could talk to each other face to face. That would be a nice change. (Beaumont, 2000, p. 162)

C'est dans un tel contexte que se tisse l'univers des trois romans e-pistolaires qui forment le corpus étudié, romans qui ont été publiés entre 2000 et 2009 et qui se déploient dans le quotidien d'entreprises de publicité et de services financiers britanniques. 


\section{@forme.roman.e-pistolaire}

Le roman e-pistolaire est entièrement écrit en mails, avec la présentation matérielle qui caractérisent ceux-ci. En effet, si le destinateur d'une lettre peut «jouer » avec les conventions liées à l'inscription du lieu et de la date, celui d'un courriel est entièrement tributaire du logiciel de messagerie qu'il emploie. Ainsi, la date et l'heure de l'envoi du message apparaissent automatiquement dans l'en-tête. L'auteur d'un courriel choisira par ailleurs d'inscrire l'objet de son message dans l'espace prévu à cet effet et choisira le ou les destinataires de son message, dont il inscrira l'adresse dans la case appropriée. Tous ces éléments qui précèdent le corps des messages qui forment l'intrigue du roman sont reproduits dans le roman, ce qui fait en sorte que l'intrigue repose sur un cadre temporel bien défini.

À tous les messages qui s'entrecroisent s'ajoutent parfois, comme c'est le cas d'un des romans qui sera étudié ici, des conversations sur le service de messagerie instantanée MSN (l'ancêtre de Windows Live Messenger), des échanges de messages textes, des entrées de blogues, des annonces de produits à vendre sur eBay, qui sont insérés entre les courriels et qui meublent ainsi l'entre-deux des messages, ajoutant alors une dimension de « direct » à l'échange e-pistolaire qui, lui, est (très légèrement) décalé dans le temps. L'intrigue progresse donc également à travers d'autres manifestations textuelles dont l'utilisation montre bien l'évolution des technologies entre 2000 , l'année de publication du premier tome du roman de Beaumont qu'on abordera, où il n'était fait usage que de courriels, et 2010, qui marque la parution du second, dans lequel le Blackberry et les services de messagerie instantanée occupent une place prépondérante. Tous ces microtextes sont 
reproduits dans la forme dans laquelle ils sont généralement représentés sur l'écran des dispositifs technologiques qui leur servent de support.

\section{@corpus}

L'intrigue de $E$ (2000) et de $E^{2}$ (2009) de Matt Beaumont ainsi que celle de Eleven (2006) de David Llewellyn sont imaginées sur un fond d'échange de trivialités entre patrons et employés : description des activités du dernier week-end, rumeurs sur des liaisons potentielles entre collègues, conversations autour du barbecue, rencontres fortuites dans un ascenseur, jalousie entre deux femmes qui tentent de séduire le même collègue, impatience des dirigeants à l'égard de leur assistante personnelle, bref, des sujets de conversation qui font contraste avec les impératifs professionnels du milieu et du contexte desquels ils émergent.

$@ E$

$E$ se déroule dans les bureaux londoniens d'une agence de publicité, Miller Shanks, qui possède aussi des succursales aux États-Unis et en Finlande. Miller Shanks «London » est engagée dans une lutte pour l'obtention du contrat de publicité de CocaCola. Mais voilà que tous les courriels de son dirigeant, David Crutton, sont automatiquement envoyés en copie conforme invisible à son homologue finlandais, qui décide aussi de se mêler du concours. Crutton exprime alors son mécontentement au service IT : 
David Crutton - 1/4/00, 8:09am

to: Chandra Kapoor

cc:

re: $\mathrm{P} 45^{\prime} \mathrm{s}$

Yesterday lunchtime I informed you that my e-mails were misrouting to Finland. I expected an immediate response. So far, sweet fuck all. I've met plumbers more reliable than your department. Do you actually want to end up washing windshields on the Mile End Road?

(Beaumont, 2000, p. 26)

La multiplication de ce type d'épisodes dans le roman permet d'illustrer ce qu'on pourrait appeler la «partageabilité » du contenu qui caractérise le courriel; autrement dit, la dimension d'instantanéité que comporte l'envoi d'un mail et le fait qu'il soit soumis à un dispositif qui peut comporter des failles techniques (comme l'envoi à des destinataires non identifiés comme tels par le destinateur du message) accentue l'absence de confidentialité liée à ce mode de communication. Or, malgré cela, patrons et employés de Miller Shanks poursuivent l'envoi massif et insouciant de plusieurs dizaines de messages par jour.

$E$ repose en outre sur les relations de pouvoir entre Crutton, ses assistantes personnelles et les membres de son équipe de production; ceux-ci essaient tant bien que mal, par toutes sortes de manœuvres toutes plus déloyales les unes que les autres, de gravir les échelons dans l'organigramme de la compagnie et de jouer un rôle plus important dans la campagne de Coca-Cola, alors que la division IT montre à plusieurs reprises toute son incompétence en étant incapable de freiner l'envoi de messages hautement confidentiels à l'ensemble des employés des multiples divisions de Miller Shanks établies à travers le monde. Tout cela se déroule pendant que le jeune Liam O’Keefe, récemment embauché par la firme, entretient 
simultanément une liaison avec deux assistantes de Crutton, Lorraine et Katie, qui l'invitent l'une et l'autre à les rejoindre tantôt dans l'ascenseur, tantôt dans le bureau d'un autre employé qui possède un sofa confortable...

Lorraine Pallister - 1/12/00, 10:44am

to: Liam O'Keefe

cc:

re: busy?

On my way in this morning I found out how to make the lift stop between floors. When are you free for elevator maintenance?

Liam O’Keefe - 1/12/00, 10:46am

to: Lorraine Pallister

cc:

re: busy?

I've got ten press ads to draw up and accounts are screaming for my time sheets ... see you in thirty seconds.

(Beaumont, 2000, p. 207)

... et que Nigel Godley, du département «Accounts», passe le plus clair de ses journées à inonder les boîtes courriels de tous les employés par des annonces de logements à louer et de produits usagés à vendre, tentant, par la formulation de slogans minables et par l'emploi de points d'exclamation superflus, de faire la démonstration de son immense potentiel publicitaire :

Nigel Godley - 1/4/00, 8:43am

to: All Departments

cc:

re: for sale

BREVILLE WAFFLE IRON

- Jasmine yellow finish

- Nearly new 
- Includes adapter to make perfect toastie sandwiches!

- The perfect way to "toast" the new Millenium!!!

- First to see will buy

- $£ 12$ o.n.o.

Call x4667 - Nige

(Beaumont, 2000, p. 27)

L'usage que font Liam O'Keefe et Nigel Goodley du courrier électronique, on le remarque ici, est une représentation éloquente de la manière dont ce qui devrait pourtant être utilisé comme un outil de travail est constamment détourné à des fins personnelles (notamment afin de se livrer à une campagne d'autopromotion auprès de ses collègues).

Cela dit, l'intérêt de $E$ réside surtout dans sa conclusion : le roman se termine en effet sur un épisode qui illustre bien les dangers et les limites du web. Alors que patrons et employés de Miller Shanks, qui a finalement réussi à décrocher le contrat de Coca-Cola, sont euphoriques, l'entreprise leur annonce que celui-ci leur est retiré parce que des membres de Miller Shanks ont été aperçus dans des vidéos compromettantes diffusées sur YouTube (dont certains qui se sont même vantés, dans des messages qui ont circulé plus tôt dans la journée, de leurs exploits éthyliques) :

Kelly Derriger / k_derriger@cokeGB.com - 1/18/00, 11:13am to: david_crutton@millershanks-london.co.uk cc: james_f_weissmuller@millershanks-ny.com re: change of circumstances

[...] I told him about our new advertising agency and to show his computer skills he keyed in Miller Shanks and executed an Internet search.

The result of this was a short clip. It featured your Executive Creative Director and a transvestite performing an act of unspeakable depravity in the very office I was given a tour of yesterday afternoon. 
[...] A brand like Coca-Cola, with its proud history of clean living and family values, could never be placed into trust of those who maintain such libertine moral standards.

It is therefore with sadness that I inform you we have decided not to award our advertising account to Miller Shanks after all.

[...] If I may close on a personal note, it sickens me to the marrow that I have been exposed to the perverted antics that you allow to go in your offices.

(Beaumont, 2000, p. 341-342)

Encore ici, le roman réussit à mettre en lumière l'un des pièges d'Internet, des réseaux sociaux et du courrier électronique qui est celui de la viralité potentielle des contenus. Celle-ci, comme il est possible de le remarquer dans le cas du contrat perdu par Miller Shanks, peut entraîner des conséquences très graves et porter atteinte à la réputation professionnelle et personnelle d'individus dont le comportement avait été jusque-là perçu comme irréprochable.

\section{$@ E^{2}$}

Dans $E^{2}$, une série d'événements viennent perturber le quotidien des employés et des patrons de la compagnie. David Crutton est désormais directeur de l'agence Meercat360, où quelques employés de Miller Shanks l'ont suivi. Les premières pages du roman sont consacrées à un message envoyé en décembre par Janice Crutton, la femme de David, aux membres de leur famille élargie, message qui vient montrer à quel point on peut raconter absolument n'importe quoi dans un courriel, qu'on peut y enrober, y déguiser, y manipuler la vérité :

Let's start with the head of the household. David has thrown himself into his new job with abandon. In fact, he's at the office now - he's slowing down a little these days, but he's still of the 
opinion that God was a slacker for taking Sunday off. [...] he has managed to find time to take up meditation. It really has transformed his outlook and he is sweetness and light personified.

(Beaumont, 2010, p. 9)

Les messages suivants montrent pourtant que Janice, une avocate de quarante-cinq ans, d'ailleurs complètement absente du premier roman, pense tout autre chose de son mari. Le courriel, tout comme la lettre, devient ainsi un espace privilégié où se réinventer, où se représenter (ainsi que sa famille) sous une persona qui est quelque peu décalée par rapport à la réalité. Janice annonce par ailleurs par courriel, dès le retour de la pause des Fêtes, qu'elle est enceinte, alors que la relation du couple bat de l'aile depuis plusieurs années :

From : Janice Crutton

To: David Crutton

Sent: 7 January 2009, 11.43

Subject: Sorry. Again

Over half an hour ago I told you I'm pregnant. No response. What the hell have you been doing? I'm going out of my mind here.

(p. 80)

David et Janice échangent ensuite quelques courriels banals sur le sujet: "I thought you were on the pill. »; «I stopped taking the pill when our sex life whittered to virtually nothing in 2005.» (p.81); «Have you thought about your options?» (p.82) Puis Crutton se retire de l'échange du moment en prétextant la présence d'un client et demande, toujours par courriel, à son assistante de ne plus répondre aux appels téléphoniques de sa femme. Cela donne le ton au reste du roman, où il s'agira pour Crutton de feindre de vouloir rester en contact avec sa femme en demandant aux autres de répondre à 
celle-ci, prétextant un emploi du temps trop chargé, tout en adoptant tous les moyens nécessaires pour garder sa femme à distance. Cette dynamique de présence-distance est justement celle qui a été évoquée en introduction. La lettre (le courriel, dans le cas qui nous intéresse ici) repose en effet sur le paradoxe selon lequel on donne à l'autre l'illusion de sa présence en déployant en même temps toute une rhétorique qui permet de repousser les prochaines interactions ou rencontres.

Par ailleurs, lorsque le fils adolescent du couple Crutton, Noah, fait une fugue, au même moment où leur fille de 15 ans est hospitalisée d'urgence, David s'en remet une fois de plus à son assistante (et aux mails), car il a des dossiers plus urgents à régler :

From: David Crutton

To: Dotty Podicra

Sent: 26 January 2009, 14.27

Subject: Family stuff

Send flowers for Tamara to all west London hospitals (not sure which one she's in). And put out an APB for Noah.

(p. 374)

Or, Dotty engage aussitôt une conversation MSN avec une amie, à qui elle demande ce qu'est un $\mathrm{APB}$, après en avoir tenté le téléchargement sur iTunes, ayant cru qu'il s'agissait d'une forme de remix. La conversation dévie rapidement vers des propos triviaux sur des collègues masculins et sur la possible homosexualité de l'un d'entre eux. La multiplication des dispositifs et la juxtaposition des échanges contribuent de manière assez éloquente à une dilution de l'information et à une confusion, encore ici, entre les tâches qui devraient être 
accomplies dans un cadre professionnel et des conversations banales sur des sujets appartenant au registre personnel.

Quant à Liam O'Keefe, qu'on a connu dans le premier roman, il croule désormais sous les dettes et entreprend de voler tout ce qui est susceptible d'être revendu (le champagne prévu pour célébrer l'obtention du dernier contrat, le nouveau photocopieur, les articles de bureau, les crayons, le papier, etc.) Il publie ensuite des dizaines d'offres de produits sur eBay. Une enquête sur ces vols, dévoilée par un chassé-croisé de messages entre les employés, meuble ainsi une partie de l'intrigue.

Parmi les personnages, on trouve enfin le stéréotype de l'idiot, Harvey Harvey, qui, inondé, tout comme ses collègues, d'un nombre considérable de courriels au quotidien, ne sait visiblement pas distinguer - comme aucune forme de tri ou de filtrage n'est pratiquée - ceux qui revêtent une certaine importance et ceux qui sont plutôt des pourriels (ou messages indésirables). Il reçoit notamment de nombreux mails à caractère pornographique comme celui-ci :

From: Britney

To: Harvey Harvey

Sent: 8 January 2009, 11.04

Subject: let's get it on

I am lonely sexual teen who need friendly chat. Reply me and we make fun. britney@gotmail.com. My breasts are on fire.

(p. 115)

Harvey H. s'empresse de répondre, ayant résolu, comme le Mychkine de Dostoïevski, "d'être courtois et sincère avec tout le monde » (Dostoïevski, 1953, p. 131) : 
From: Harvey Harvey

To: Britney

Sent: 8 January 2009, 11.06

Hi Britney

Amazing that you should write because I've only just had an email from another girl who was bored and wanted to chat. She's called Daniela and she seems very nice. Remarkably, she also complained of inflammation in her breasts. Maybe it's a winter virus thing. I really think you should get in touch with her. I'm sure you'd cheer each other up. Her e-mail is daniela@gotmail.com

Thanks for writing. And do get in touch with Daniela. I bet it helps chase away the January blues.

Harvey Harvey

(Beaumont, 2010, p. 116)

H. H. se rend même en Afrique pour sauver une jeune Nigériane dont les ravisseurs exigent une rançon importante. Ce qui fait la singularité de ce personnage est son authenticité ainsi que le fait qu'il agisse sous la seule impulsion de sa conscience et de son empathie pour le reste de l'humanité, et non pas en fonction de ce que les autres lui dictent. Loin d'être imbécile à proprement parler, l'idiot, auquel on associe ici le personnage d'Harvey, est, comme l'écrit Pierre Truchot, "l'être qui agit de lui-même, qui, de sa propre initiative, pense, se comporte, voire crée en fonction de règles qu'il s'est lui-même prescrites. [...] l'idiot ignore, les codes, us et coutumes de la société dans laquelle il vit.» (T2010, p.1) Cela signifierait donc que le courriel possède son propre code et qu'il appartient à un imaginaire singulier que l'idiot, ne possédant pas la capacité de discernement nécessaire à un tel exercice, est incapable de maîtriser. 


\section{@Eleven}

Quant à Eleven, qui a été comparé, par certains critiques, à la télésérie britannique The Office (2001), dont l'intrigue met en vedette des employés de bureau d'une usine de fabrication de papier, il raconte la journée du 11 septembre 2001 chez Quantum Finance, une boîte de finance de Cardiff, en GrandeBretagne, où les employés, qui se caractérisent par leur jalousie et leur hypocrisie chroniques, semblent entretenir les uns à l'égard des autres beaucoup de méfiance. Un des éléments les plus singuliers du roman réside dans le fait que le personnage principal, Martin Davies, qui, sans détester son emploi, paraît le trouver tout à fait inutile, écrit au courant de cette journée une série de messages inachevés qu'il enregistre dans le dossier «brouillons » de son logiciel de courrier électronique, messages dans lesquels il dresse le bilan (plutôt négatif) de sa vie personnelle et professionnelle :

FROM: martin.davies@quantumfinance.co.uk

TO:

SAVED: 10:15, Tuesday September 11, 2001

SUBJECT: Break

In the car park there are two fenced off spaces that have been allocated to the smokers and that's where we stand.

There's one guy who's always smiling. He's always on his own, and he's always smiling to himself, as if he's about to start laughing. I feel so nervous around people like that. I wonder was he always like that or has it happened since he started working here?

I'm beyond bored. Boredom would be comforting right now.

This is something else. This is everything.

(Llewellyn, 2006, p. 28)

Ces messages, mis les uns à la suite des autres, forment une sorte de journal introspectif qui fait contraste avec le chassé- 
croisé de courriels banals échangés par les employés de la compagnie et qui accentue la détresse du personnage souhaitant changer de trajectoire de vie: certains de ces courriels «brouillons » sont en effet implicitement destinés à un éditeur auquel Martin Davies propose un roman qu'il vient de terminer. Alors que $E$ et $E^{2}$ associent le potentiel créatif à la figure de l'idiot, on trouve, dans Eleven, un personnage beaucoup plus sérieux, qui a une certaine épaisseur psychologique, qui occupe certes une position enviable dans la hiérarchie de l'entreprise, mais qui veut fuir ce monde factice. C'est par l'écriture qu'il tente de se sortir de son désespoir; après le cliché de l'idiot, nous avons ainsi celui de l'individu à la fois tourmenté et blasé, pour qui l'écriture représente une sortie de secours. Il est de surcroît paradoxal que les seuls messages comportant un véritable contenu soient ceux qui restent sans destinataire.

Davies est toutefois le seul personnage qui évolue véritablement dans le roman. Vers le milieu de l'après-midi, l'annonce des attentats contre les tours jumelles et contre le Pentagone aurait, selon toute logique, dû entraîner un changement de registre dans les messages échangés entre les employés de Quantum Finance. Or, ce n'est pas du tout ce qui se produit. Plusieurs des personnages ont d'ailleurs consommé drogues et alcool pendant la journée, si bien qu'on comprend rapidement qu'ils ne sont pas tout à fait conscients de l'importance et de la gravité de la tragédie qui est en train de se produire aux États-Unis.

FROM: Martin Davies

(mailto: martindavies@quantumfinance.co.uk)

T0: lloydt@callotech.co.uk

SENT: 15:36, Tuesday September 11, 2001 
SUBJECT : RE:

Lisa told me about the barbecue.

FROM: Lloyd Thomas

(mailto: lloydt@callotech.co.uk)

T0:martindavies@quantumfinance.co.uk

SENT: 15:36, Tuesday September 11, 2001

SUBJECT: RE: RE:

Oh.

FROM: Martin Davies

(mailto: martindavies@quantumfinance.co.uk)

T0: lloydt@callotech.co.uk

SENT: 15:37, Tuesday September 11, 2001

SUBJECT: RE: RE: RE:

Is it possible for you to meet a woman without $\mathrm{f}^{* *}$ king them?

(p. 107)

Eleven montre en somme, de manière éloquente - et tout à fait caricaturale -, l'ennui et la frustration d'individus qui occupent un emploi routinier qui ne répond pas tout à fait à leurs aspirations et qui paraissent dès lors évoluer dans un monde parallèle, loin des préoccupations habituelles et, surtout, de la retenue qu'impose la vie professionnelle.

\section{@banalité.incommunicabilité}

$E, E^{2}$ et Eleven reposent ainsi tous trois sur des récits banals d'individus sans envergure, dont le quotidien, sans importance, ressemble étrangement à celui de tous les travailleurs de grandes entreprises à travers le monde occidental. Ils illustrent aussi, d'une manière particulière, l'un des plus grands topoï du genre romanesque depuis le XIXe siècle : l'incommunicabilité. 
Celle-ci est remarquée non seulement dans le couple, où elle s'accompagne alors d'une sorte de fatalité (on pense ici aux Crutton dans $E^{2}$ ), mais aussi dans la manière dont les employés communiquent entre eux.

À cet égard, la forme du roman par courriels introduit un autre paradoxe intéressant : on écrit généralement à quelqu'un pour dire quelque chose. Or, dans les trois cas qui nous intéressent ici, les messages ne contiennent à toutes fins pratiques que des bruits de fond, des rumeurs insignifiantes; ils se font le reflet d'un grand vide existentiel. Même les messages envoyés à l'ensemble des employés sont reçus dans l'indifférence la plus totale. On reçoit tellement de courriels qu'on les place tous au même rang d'importance avant même de les avoir lus. Un des meilleurs exemples de cette indifférence reste un courriel annonçant un exercice d'évacuation de l'immeuble dans $E^{2}$, message qui est ignoré par la haute direction qui choisit de faire fi de la directive officielle et de tenir tout de même la réunion du conseil d'administration à ce moment.

Toute cette accumulation de banalités contribue pourtant à la construction de véritables romans. Les personnages et les dynamiques qui sous-tendent les relations, notamment ce paradoxe de présence et de distance évoqué d'entrée de jeu et qu'il a été possible d'identifier comme un élément clé des échanges, se révèlent au fur et à mesure que les chassés-croisés de messages sont déclinés. Progressivement, on découvre des personnages qui ont tous une personnalité singulière (malgré les situations très caricaturales dans lesquels ils sont transposés), des personnages qui ont chacun un passé, une histoire et, surtout, qui ont des aspirations, tant 
professionnelles que personnelles. Malgré l'apparence de banalité et d'inutilité de la plupart des courriels échangés, malgré la pléthore de non-événements qui ponctuent les messages, chacun des personnages se construit en effet progressivement par rapport aux autres personnages avec lesquels il interagit, par le ton qu'il emploie, par la structure de phrases qu'il adopte, par son vocabulaire particulier, par les signes typographiques auxquels il recourt de manière récurrente (comme en témoigne l'exemple des points d'exclamation employés de manière exponentielle cité précédemment). Ces récits, révélés de façon fragmentaire, comportent par ailleurs une large part de non-dit, qui fait en sorte de conserver l'intérêt du lecteur jusqu'à la fin. Il y aurait ainsi, sous cette première couche de trivialités qu'il semble impossible de franchir, une certaine substance qui, c'est du moins ce que la forme des messages et la nature des dispositifs de transmission suggèrent, ne paraît cependant plus pouvoir être communiquée.

\section{@banalité.quotidien}

Il resterait à réfléchir à ce qui se cache sous cette banalité : pourquoi, malgré l'abondance d'information qui circule dans les courriels, reste-t-on néanmoins dans ce registre? Est-ce le média qui l'impose? Est-ce justement, comme on vient de le suggérer, la forme à laquelle il contraint l'utilisateur qui finit par générer la banalité? Est-ce la surcharge de messages qui finit par entraîner, comme le prétendait déjà Jean-Pierre Balpe en 1997 dans un entretien accordé à Pierre Lévy pour le journal Le Monde, une absence de réflexion, de laquelle découlerait 
justement la banalité2? Ou celle-ci serait-elle plutôt rattachée à un malaise plus grave qui affecterait la société actuelle? Rappelons que, dans les trois romans évoqués, les seuls mails qui présentent une certaine profondeur sont ceux enregistrés par Martin Davies dans le dossier «brouillons » et qui, dès lors, ne sont pas destinés, du moins pas dans un premier temps, à être lus par autrui. Que cela révèle-t-il sur l'omniprésence des systèmes de messageries instantanés, du courriel, des autres plateformes et dispositifs permettant de rester branché en tout temps? On a demandé à Marc Beaumont, dans une entrevue en août 2009, si ses livres, qui évoquent notamment Big Brother, YouTube, des blogues étranges, par exemple, sont justement le reflet de la perplexité qu'il entretient à l'égard du monde actuel. L'auteur a alors précisé que, selon lui, Internet est le miroir d'une folie collective : « No bemusement. The internet - blogs, YouTube, Facebook, all of that limitless, unedited, selfaggrandising shite - holds up a perfect mirror to our insanity. The modern world is fucked, innit? Thank the lord, because that's where all the comedy is. " (Brew, 2009) À en croire Beaumont, les dispositifs de communication et de publication virtuels nous renverraient donc le reflet de ce que nous sommes devenus. Serions-nous alors tous des Harvey Harvey? Serionsnous, à divers degrés, des « idiots » qui ne maîtrisent pas encore tous les codes sémantiques et sociaux liés à l'usage de tels dispositifs, mais qui sont tout de même conscients de leur potentiel inventif et qui en font (dans certains cas) un usage innovant?

\footnotetext{
2 «Avec le courrier électronique, vous avez à peine le temps de répondre à une question qu'une autre arrive. La compression du temps réclame [pourtant] une gestion de la réflexion. » (Lévy et Balpe, 1997)
} 
Le roman par courriels n'est d'ailleurs pas la seule manifestation littéraire à reposer sur la banalité du quotidien. Dans l'introduction à un dossier consacré à la banalité dans la littérature contemporaine, Sandrina Joseph rappelle que celle-ci touche une diversité de genres - l'autofiction, le roman et l'essai, par exemple - et qu'elle caractérise tout aussi bien la production européenne que la littérature nord-américaine: "La lisibilité du texte contemporain semble [...] se fonder sur l'évocation de l'expérience la plus manifestement commune, [...] comme si l'emprise de l'usé sur nos vies valait à ce point la peine d'être consigné. » (2009, p.13) Pourquoi cette banalité nous intéresse-t-elle autant? Qu'est-ce que cette mise en valeur de la trivialité, de l'insignifiant, vient combler? Bruce Begout, dans La Découverte du quotidien, rappelle à cet égard que

nous assistons, ébahis et fascinés, à des activités ménagères sans intérêt, [...] à des conversations anodines sur des sujets éculés, et, loin d'être contrarié ou irrité, l'on se console ou se réjouit, en se disant que tout ça, [...] les paroles ordinaires et les poncifs, les gestes habituels et sans grâce, c'est nous, c'est nous qui l'avons fait et le faisons tous les jours, ce prodige éternellement recommencé de la banalité quotidienne. (2005, p. 24; l'auteur souligne)

C'est peut-être l'une des raisons pour lesquelles Harvey Harvey est aussi fascinant. Personnage en apparence le plus insignifiant des trois romans abordés, celui-là même qui croit qu'il existe un virus hivernal s'attaquant aux seins des femmes et qui a volé au secours d'une jeune Nigériane demandant des centaines de milliers de livres, en arrive à la conclusion suivante au sujet des courriels : "I love e-mail. [...] I had an amazing thought this morning. You could tell a whole story just by using emails. Imagine an entire novel where you're peeking into people's private inboxes. Wouldn't that be brillant? » (Beaumont, 2010, 
p. 170) Harvey Harvey, qui incarne la naïveté et l'innocence en puissance et qui croit en la bonté de l'humanité tout entière, est pourtant le seul qui propose, en voyant dans l'assemblage éventuel de tout ce discours vide de sens généré au quotidien par les courriels un réservoir riche pour la rédaction d'un roman, une réflexion intéressante sur la création. En effet, dans cet environnement d'agence de publicité où tous les employés sont engagés au quotidien dans une course à la productivité, Harvey Harvey montre que l'idiot est finalement susceptible d'être le plus créatif de tous. Les trois romans illustrent ultimement l'inaptitude de l'individu contemporain à distinguer le vrai du faux, l'important du banal, ce qui se reflète de façon marquée dans les nouvelles formes d'expression et de communication où la représentation qu'on offre de soi notamment dans les milieux professionnels - devient le reflet de cette incapacité. 


\section{Bibliographie}

BEAUMOnT, Matt. (2000), E, Londres, Plume.

—. (2010 [2009]), E2, Londres, Plume.

Begout, Bruce. (2005), La Découverte du quotidien, Paris, Alia.

BISMUTH, Nadine. (2004), Scrapbook, Montréal, Boréal.

BREW, Simon. (2009), « Matt Beaumont Interview », Den of Geek, 14 août, <http://www.denofgeek.com/books-comics/11392/mattbeaumont-interview $>$.

DostoÏEVSKI, Fédor. (1953 [1869]), L'Idiot, Paris, Gallimard, coll. «Folio ».

Glattauer, Daniel. (2010), Quand souffle le vent du nord, Paris, Grasset, coll. « Le Livre de poche ».

JoSEPH, Sandrina (dir.). (2009), « Révéler l'habituel. La banalité dans le récit littéraire contemporain », Paragraphes, vol. 28.

KaUfmann, Vincent. (1990), L'Équivoque épistolaire, Paris, Minuit, coll. « Critique».

LAGRAÑA, Fernando A. A. (s.d.), «Les sept péchés capitaux du mail», <http://www.itu.int/ITU-D/membership/docs/7PechesFrench.pdf>.

LÉVY, Pierre et Jean-Pierre BALPE. (1997), « [entretien] », propos recueillis par Michel ABERGANTI, supplément, Le Monde, 20 novembre, <http://www.site-magister.com/bts/synthese3b.htm>.

LLEWELLYN, David. (2006), Eleven, Wales, Seren Books. 
MELANÇON, Benoît. (2010), «Épistol@rités, d'aujourd'hui à hier», Lumen: Selected Proceedings from the Canadian Society of Eighteenth-Century Studies / Lumen : travaux choisis de la Société canadienne d'étude du dix-huitième siècle, vol. 29, p. 1-19.

Rushton, Rosie et Nina Schindler. (2000), P.S. He's Mine!, Londres, Piccadilly Press.

TRUCHOT, Pierre. (2010), « Figures de la singularité: l'idiot et ses rythmes», Rhuthmos, 11 novembre. <http://rhuthmos.eu/spip.php?article206>.

WACHEndoRfF, Martina. (2001), Le Baiser électrique, Paris, Gallimard, coll. « NRF ». 


\title{
Résumé
}

Le roman par courriels, contrairement au roman épistolaire traditionnel (dans lequel les protagonistes écrivent surtout pour se mettre en valeur), paraît reposer sur une dynamique d'échange de considérations des plus banales. Dans $E, E^{2}$ et Eleven, qui se déploient en entreprise, cet échange de trivialités devrait normalement laisser place à l'adoption d'un registre plus sérieux lorsqu'un événement grave survient. L'article examine notamment la pléthore de courriels vides de sens qui forment l'intrigue de ces trois romans en tentant de mettre en lumière ce que révèlent la superficialité et la banalité sur l'individu et la société de notre siècle.

\begin{abstract}
If in the traditional epistolary novel the protagonists mostly write letters to showcase themselves, in a scope of both seduction and confrontation, the " $e$-pistolary» novel (by email) seems to be based on a dynamic of considerate and mundane exchanges. In $E, E^{2}$ and Eleven -, novels whose plot unfold against a corporate backdrop, the exchange of trivialities would normally make way for a more serious tone when problems emerge or major events occur. We will try to expose, by examining this plethora of meaningless emails, what can be revealed about the individual and society of our time through the superficiality and banality of those exchanges.
\end{abstract}

